\title{
A Comparative Study of Ziehl-Neelsen Stain and Fluorescent Stain Microscopy in the Diagnosis of Pulmonary Tuberculosis
}

\author{
Hajra Tasneem Masali $(\mathbb{D}$, Aparna Takpere* (D) and Praveen Shahapur (D) \\ Department of Microbiology, Shri BM Patil Medical College, BLDE (Deemed to be University) \\ Vijayapura - 586 103, Karnataka, India.
}

\begin{abstract}
Tuberculosis remains a global disease, killing over 2 million people each year. India accounts for twothird of the global total i.e. $26 \%$. A good, fast, and economical test to diagnose a disease is urgently needed so that effective treatment can begin as soon as possible. Various investigations can be done for TB diagnosis and these include clinical suspicion, Chest imaging, acid-fast bacilli staining, MTB culture, serological methods and Assays for amplification of nucleic acids. For developing countries like India due to the enormous number of patients and budget restrictions, the assessment of a quick and low-cost diagnostic procedure is critical. A present prospective study was undertaken to compare Ziehl-Neelsen stain with Fluorescent stain microscopy in detecting of Mycobacterium tuberculosis in TB patient's sputum samples. Among 274 sputum samples collected from suspected TB cases, 50 (18.2\%) were positive by ZN and 116 (42.3\%) were positive by fluorescent microscopy. The sensitivity, specificity, Positive predictive value (PPV) \& Negative predictive value (NPV) of FM microscopy was $98 \%, 70 \%$, $42 \%$, and $99 \%$ respectively. FM microscopy was superior to $\mathrm{ZN}$ microscopy in detecting pulmonary tuberculosis cases as it could detect $\mathbf{2 4 . 1 \%}$ more positive cases than $\mathrm{ZN}$ staining. Fluorescence staining provides better sensitivity and specificity and can detect accurately more paucibacillary cases hence is of diagnostic value and can help in the early treatment of pulmonary tuberculosis.
\end{abstract}

Keywords: Mycobacterium tuberculosis, Ziehl-Neelsen staining, Fluorescent staining

*Correspondence: aparnaytakpere@gmail.com; +91 8050439036

(Received: May 21, 2021; accepted: September 29, 2021)

Citation: Masali HT, Takpere A, Shahapur P. A Comparative Study of Ziehl-Neelsen Stain and Fluorescent Stain Microscopy in the Diagnosis of Pulmonary Tuberculosis. J Pure Appl Microbiol. 2021;15(4):2027-2033. doi: 10.22207/JPAM.15.4.24

(C) The Author(s) 2021. Open Access. This article is distributed under the terms of the Creative Commons Attribution 4.0 International License which permits unrestricted use, sharing, distribution, and reproduction in any medium, provided you give appropriate credit to the original author(s) and the source, provide a link to the Creative Commons license, and indicate if changes were made. 


\section{INTRODUCTION}

Tuberculosis continues to be a significant public health concern according to Global TB report 2020. ${ }^{1}$ Mycobacterium tuberculosis infects over a third of the world's population, killing over 2 million people each year. ${ }^{2}$ According to World Health Organization (WHO) Tuberculosis infections are currently spreading at the rate of $1 \%$ per sec per million people and is a single largest cause of loss in healthy life year in productive age group. ${ }^{3}$ During the year 2020, it is expected that roughly 1 billion people would be infected with tuberculosis, 200 million will acquire the disease, and 35 million will die from it. India is one among the biggest contributors to the increase in tuberculosis in terms of estimated incident cases per year accounting for two-third of the global total i.e. $26 \%$. The situation is further worsening due to increase of drug resistant variants (MDR) of Mycobacterium tuberculosis. There is an urgent need for a good, fast, and economical test that can detect a disease so that effective treatment may begin early. ${ }^{4}$

Various investigations can be done for TB diagnosis and these include clinical suspicion, chest imaging, acid fast bacilli staining, MTB culture, serological methods and assays for amplification of nucleic acids. The conventional direct microscopy of sputum smears is foremost rapid, inexpensive, highly specific and capable of identifying the most infectious cases of Tuberculosis. It is also useful for assessing therapeutic response and determining whether a patient is cured or not at the end of treatment. ${ }^{5}$ However the main limitation of the method is its low sensitivity in developing countries especially in patients co- infected with HIV. ${ }^{6}$

Since 2011, WHO has advised using LED Fluorescence Microscopy (FM) as a phased alternative to Ziehl-Neelsen (ZN) for a faster diagnosis of tuberculosis. FM has greater benefits than $\mathrm{ZN}$ as it detects around 5-10\% more acid fast bacilli (AFB) than ZN because AFB Fluorescence has a stronger contrast than ZN (1000x), slides may be screened at a lower magnification (200x or 400x) and inspected more rapidly, thereby alleviating the excessive workload in certain laboratories. Furthermore, FM staining is less complicated; preparing the auramine solution is less difficult. And because no immersion objective is necessary to see the bacilli, there is no need to utilize the immersion oil and xylene that $Z N$ requires which are both costly and can harm the objectives owing to abuse or poor quality. However, the complexity of the microscope, the requirement for a dark room, the perceived health risk associated with UV light exposure, and the fact that mercury lamps lighting systems are relatively expensive, have a short life span, require stable electrical supply, and new bulbs may be difficult to procure are all downsides of fluorescent microscopes. ${ }^{7,8}$

The gold standard for microbiological diagnosis of tuberculosis in underdeveloped countries is culture on Lowenstein Jensen solid medium, which needs roughly 1 bacilli $/ \mathrm{ml}$ of samples for mycobacterium recovery. Due to the pathogen's delayed growth rate, a definite diagnosis might take up to 4-6 weeks. ${ }^{2}$

Serological tests are currently not reliable enough for the diagnosis of tuberculosis. ${ }^{9}$

More advanced diagnostics such as Expert MTB / Rif or Polymerase chain reaction are not available for routine tuberculosis diagnosis and also require stable uninterrupted electrical supply, ambient operating temperature of instrument, continuous monitoring of shelf life of cartridges and also needs security measures. ${ }^{10,11}$

Therefore the current prospective study was carried out to compare Ziehl-Neelsen stain with fluorescent stain microscopy in the identification of Mycobacterium tuberculosis in sputum samples from pulmonary TB patients.

\section{MATERIALS \& METHODS}

- This study was conducted in a tertiary care facility from July 2019 to January 2021 in clinically suspected MTB inpatients \& outpatients.

- The study was carried out after the Institutional Ethical Committee approved it.

- Prior to recruitment, all subjects gave their written informed approval. The records were handled in absolute confidence.

\section{Inclusion Criteria}

1. Age $>15 y$ rs

2. Having $2 /$ more of the following symptoms indicates clinically suspected pulmonary tuberculosis: Fever, persistent cough, weight loss, and an increase in temperature in the evening Pleurisy chest discomfort, hemoptysis, 
and an abnormal chest radiograph are all signs of pulmonary TB.

3. No history of receiving ATT within 3 months before enrolment

4. All presumptive TB patients infected with HIV. Exclusion Criteria

1. Age less than 15 yrs.

2. Patients with history of lung malignancies

3. Samples received without clinical history

4. All Extra pulmonary cases

- A total of 274 sputum samples were taken from individuals suspected of having pulmonary TB. After providing the patients appropriate instructions, two sputum samples: spot and early morning, were collected in a sterile, wide mouth, leak proof container. An excellent sputum specimen is comprised of freshly expectorated material with a volume of around $5 \mathrm{ml}$. The specimens were taken to the laboratory as soon as possible after being collected. The samples were processed in a BSC - 2 with all essential precautions taken.

- About $5 \mathrm{ml}$ of sputum was mixed with an equivalent volume of 4 percent $\mathrm{NAOH}$ and centrifuged at $3000 \times \mathrm{g}$ for 15 minutes to concentrate and decontaminate the sample. Following decontamination, sediment was collected, smears were prepared, and ZN and fluorescent staining were used to stain the smears.

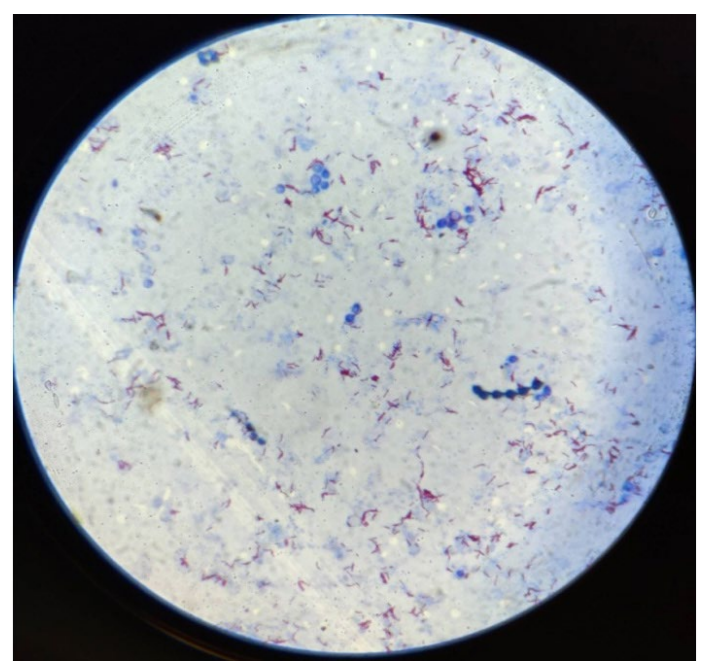

Fig. 1: Shows acid fast bacilli with Ziehl-Neelsen staining.

\section{Smear microscopy}

Ziehl-Neelsen Smear Staining

The heat-fixed smears were placed with the smear side up on the staining bridge and stained with filtered 0.3 percent carbol fuchsin. The smears were heated and colored for 5 minutes before being cleaned and drained. Then it was decolorized for 30 seconds with 25 percent sulfuric acid, washed with water, and drained. After that, they were counterstained with a $0.1 \%$ methylene blue solution for 1 minute before being washed with water. The smears were allowed to dry naturally before being viewed under the microscope with the oil immersion [100x] objective. To declare negative smear, a minimum of 100 oil immersion fields were observed.

\section{Fluorescent Smear Staining}

The heat fixed smear was flooded with filtered 0.1 percent auramine 0 for 20 minutes, then washed well with running tap water to avoid washing away the smear, and then decolorized with 0.5 percent acid alcohol for 30 seconds and washed with running tap water. They were covered with 0.5 percent potassium permanganate and counterstained for 1 minute before being washed. The smears were allowed to air dry before being viewed microscopically with a dry [40x] objective lens in an LED illumination-based Fluorescence microscopy system. Acid fast bacilli have a golden, thin, rod-shaped structure that fluoresces. ${ }^{5}$

- The NTEP 2020 guidelines were used to

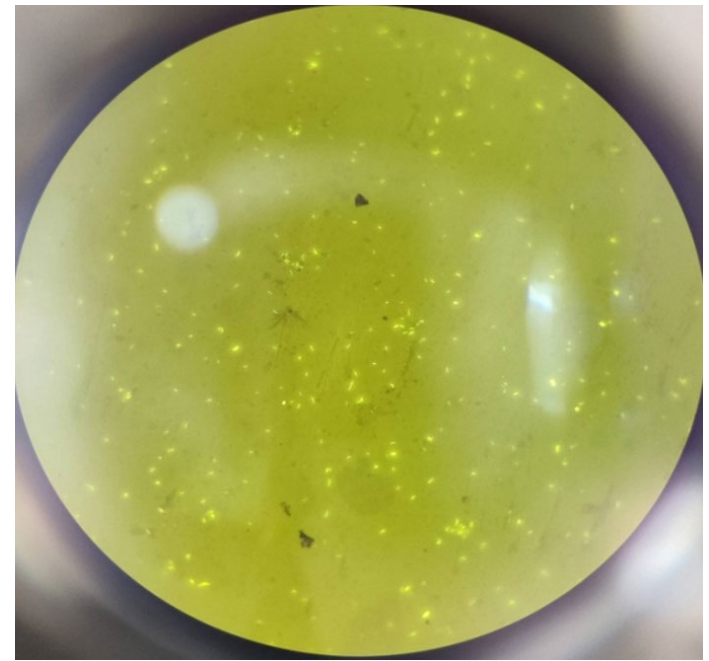

Fig. 2. Shows acid fast bacilli with fluorescent staining. 
grade acid fast bacilli (AFB) by both staining methods. ${ }^{12}$

\section{Data Analysis}

The data was entered into a Microsoft Excel spread sheet, and statistical analysis was carried out using the SPSS statistical software for social sciences (Version 21). Drawings, Mean SD, counts, and percentages were used to depict the findings.

Sensitivity and specificity, Positive predictive value, Negative predictive value, and accuracy were all calculated as diagnostic tests. The chi square test was used to compare categorical variables.

\section{RESULTS}

The present study was done for one and a half years, from July 2019 to January 2021, and a total of 274 sputum samples were collected from patients with presumptive pulmonary tuberculosis who visited our hospital.

Table 3 shows that out of 274 sputum samples, 50 (18.2\%) were positive by ZN and 116

Table 1. Distribution of age groups

\begin{tabular}{lcc}
\hline $\begin{array}{l}\text { Age in } \\
\text { Years }\end{array}$ & $\begin{array}{c}\text { Number of } \\
\text { patients }\end{array}$ & Percentage \\
\hline $16-25$ & 14 & 5.1 \\
$26-35$ & 61 & 22.3 \\
$36-45$ & 60 & 21.9 \\
$46-55$ & 45 & 16.4 \\
$56-65$ & 46 & 16.8 \\
$66-75$ & 32 & 11.7 \\
$75+$ & 16 & 5.8 \\
Total & 274 & 100.0 \\
\hline
\end{tabular}

Table 3. Comparison of ZN \& FM stain microscopy

\begin{tabular}{lcccc}
\hline & \multicolumn{2}{c}{ FM Microscopy } & Total & Chi square test \\
\cline { 2 - 3 } ZN Microscopy & Positive & Negative & & \\
\hline Positive & 49 & 1 & 50 & $\mathrm{X}^{2}=77.627$ \\
& & & & $\mathrm{P}=0.0001^{*}$ \\
$\%$ & $98.0 \%$ & $2.0 \%$ & $100.0 \%$ & \\
Negative & 67 & 157 & 224 & \\
$\%$ & $29.9 \%$ & $70.1 \%$ & $100.0 \%$ & \\
Total & 116 & 158 & 274 & \\
$\%$ & $42.3 \%$ & $57.7 \%$ & $100.0 \%$ & \\
\hline
\end{tabular}

\footnotetext{
*:Highly significant difference.
}

(42.3\%) were positive by fluorescent microscopy. With a $P$ value of 0.0001 , the result is very significant.

Table No.4 shows higher grading scores by FM method: $274[41+23+13+40]$ positive versus $100[11+20+7+12]$ positive by ZN method which was highly significant with a $P$ value of 0.0009 .

Considering ZN staining as gold standard, Table 5 shows that sensitivity, specificity, PPV \& NPV of FM microscopy was $98 \%, 70 \%, 42 \%$ and $99 \%$ respectively.

\section{DISCUSSION}

- The purpose of this study was to compare the detection of Mycobacterium tuberculosis in sputum samples of suspected pulmonary TB patients using Ziehl-Neelsen stain versus Fluorescent stain microscopy.

- In the present study we found that 26-45 years age group was commonly affected by MTB which was consistent with studies by Jadhav et al. ${ }^{13}$ which showed $21-40$ years and Lawrence et al. ${ }^{14}$ showed $18-30$ years age group as the predominantly age group affected which justifies that tuberculosis is common in economically productive age group. But Neelu sree et al, Pathirikar TG et al showed $51-60$ years and $51-70$ years as the predominant age group affected. ${ }^{2,22}$

Table 2. Gender wise distribution

\begin{tabular}{lcc}
\hline Gender & No. of patients & Percentage \\
\hline Female & 90 & 32.8 \\
Male & 184 & 67.2 \\
Total & 274 & 100.0 \\
\hline
\end{tabular}

ournal of Pure and Applied Microbiology 
- Our study revealed a high prevalence of male patients [67.2\%] than females [32.8\%]. Similar findings were found in other studies done by Neelu sree et al $^{2}$ who reported prevalence of $52.3 \%$ in Males \& $47.7 \%$ in Females, Makesh kumar et al. ${ }^{15}$ showed $59.8 \%$ in males and $40.2 \%$ in Females. The study provides strong evidence that pulmonary TB prevalence is higher in males than in females. According to the TB Annual Report, the incidence of tuberculosis is substantially greater among males than females and in older age groups. ${ }^{2}$

- In this study, fluorescent staining was shown to be 24.1 percent more efficient than ZN staining. FM showed [42.3 percent] higher degree of positivity than the ZN method [18.2 percent]. This was similar to studies like Goyal R et al showed ZN 7.47 percent, AO 14.9 percent, A.R.K Archana et al showed ZN 8.59 percent, AO 13.77 percent, $S$ Laifangbam et al showed ZN 44.1 percent, AO 71.6 percent, Namaji MA et al. showed ZN 18 percent, AO 24.67 percent, Khanna A et al showed ZN 26.5 percent, AO 35.9 percent $3,4,16,17,18$. According to this study fluorescent staining of sputum smears is a better method of microscopy for identifying pulmonary tuberculosis patients than ZN staining. Soham Gupta et al. did a study in

Table 4. Difference in grading score

\begin{tabular}{lccc}
\hline Grading & $\begin{array}{c}\text { FM Grading } \\
\text { No. of } \\
\text { patients (\%) }\end{array}$ & $\begin{array}{c}\text { ZN Grading } \\
\text { No. of } \\
\text { patients (\%) }\end{array}$ & $\begin{array}{c}\text { Chi } \\
\text { square } \\
\text { test }\end{array}$ \\
\hline $1+$ & $41(15.0)$ & $11(4)$ & $\mathrm{X}^{2}=16.503$ \\
& & & $\mathrm{P}=0.0009^{*}$ \\
$2+$ & $23(8.4)$ & $20(7.3)$ & \\
$3+$ & $13(4.7)$ & $7(2.6)$ & \\
Scanty & $40(14.6)$ & $12(4.5)$ & \\
Total & 274 & 100.0 & \\
\hline
\end{tabular}

${ }^{*}$ Highly significant difference.

Table 5. FM VS ZN microscopy Sensitivity, Specificity, Positive predictive value (PPV) \& Negative predictive value (NPV)

\begin{tabular}{lccccr}
\hline Diagnostic test & Sensitivity & Specificity & PPV & NPV & Accuracy \\
\hline FM VS ZN & $98 \%$ & $70 \%$ & $42 \%$ & $99 \%$ & $75 \%$ \\
$95 \%$ Cl & $89 \%$ TO 99\% & $64 \%$ TO 76\% & $33 \%$ TO 52\% & $97 \%$ TO 99\% & \\
\hline Journal of Pure and Applied Microbiology & & 2031 & & www.microbiologyjournal.org
\end{tabular}

Karnataka in 2010 that found the least [0.4] fluorescence positivity when compared to ZN positivity. ${ }^{23}$

- In our study, ZN staining technique had a higher percentage of false negatives (29\%) than FM staining technique (2\%), which was similar to studies by Eben et al who found ZN staining technique false negatives (45.2\%) and FM staining technique false negatives (15.5\%) 5 And Sinamangal et al who found ZN staining technique false negatives (40.27\%) and FM staining technique false negatives (40.27\%) 19 As a result, a high number of cases overlooked by ZN staining were easily discovered by fluorescent staining.

- The findings of this study indicates higher grading scores by FM : $274[41+23+13+40]$ positive versus $100[11+20+7+12]$ positive by $\mathrm{ZN}$ method, which is comparable to the study by Roma et al FM score of 57[10+47] and ZN score of 29[4+25], Archana et al FM score of $93[13+24+22+34]$ and $Z N$ score of $58[2+10+18+28]$, Khanna et al FM score of $84[9+19+22+34]$ and ZN score of $62[3+7+20+32]$, Golia et al FM score of $105[18+8+35+44]$ and ZN score of $67[1+2+2+36] .^{3,4,18,21}$

- The sensitivity, specificity, positive predictive value, and negative predictive value of $\mathrm{FM}$ staining were estimated using $Z N$ staining as the gold standard. Our study showed sensitivity \& specificity of FM as 98 percent and 70 percent respectively. This higher sensitivity was confirmed with other studies done by Dzodanu et al (Sensitivity 84.5 percent, Specificity 100 percent), Kumar et al (Sensitivity 95.45 percent, Specificity 99.45 percent), Namaji MA et al (Sensitivity 86.84 percent Specificity 96.43 percent), Bhansal et al (Sensitivity 85 percent, Specificity 96 percent). 5,15,17,20 FM appears to be a more sensitive approach than $\mathrm{ZN}$ because as it 
can detect low bacillary load in sputum, has a higher absorbability of Mycolic acid by auramine than fuchsin, and has a broader field area to examine. And decreased specificity of Led FM for AFB might be explained in part by a lack of expertise or training among technicians.

- $\quad$ Present study showed PPV \& NPV of $42 \%$ and $99 \%$ respectively. This was similar to other studies done by Neelu et al. (PPV- 84.4\% and NPV-100\%) and Laifangbam et al (PPV 94.59\% and NPV- 92.86\%). ${ }^{2,16}$ False positive smears with FM can be caused by impurity in the auramine, food particles, and artifact that create fluorescence; hence all scanty and suspicious cases should be verified with ZN staining.

\section{CONCLUSION}

Overall our data showed that FM staining microscopy was better than ZN staining microscopy method in identifying pulmonary $\mathrm{TB}$ patients, with FM microscopy identifying 24.1 percent more positive cases than ZN staining. And also fluorescence staining approach has higher sensitivity and specificity, and can properly detect more paucibacillary cases, making it useful for diagnosis and early treatment of pulmonary TB.

\section{ACKNOWLEDGMENTS}

None.

\section{CONFLICT OF INTEREST}

The authors declare that there is no conflict of interest.

\section{FUNDING}

None.

\section{AUTHORS' CONTRIBUTION}

All authors listed have made a substantial, direct and intellectual contribution to the work, and approved it for publication.

\section{DATA AVAILABILITY}

All data generated or analyzed during this study are included in the manuscript. institutional ethical committee of Shri BM Patil medical college hospital and research Centre and the study was conducted in Mycobacteriology division of department of microbiology.

\section{REFERENCES}

1. World Health Organization. (2018). Global tuberculosis report 2018. World Health Organization. https://apps. who.int/iris/handle/10665/274453. License: CC BY-NCSA 3.0 IGO

2. Neelu SP, Terin J, Dutta H, Kalyani M. Comparison of Ziehl-Neelsen, Kinyoun's and Fluorescent Staining for detection of Mycobacterium tuberculosis in sputum samples before and after petroff's concentration technique. Asian J Pharm Clin Res. 2018;11(4):110-113. doi: 10.22159/ajpcr.2018.v11i4.23662

3. Goyal R, Kumar A. A comparison of Ziehl-Neelsen staining and fluorescent microscopy for diagnosis of pulmonary tuberculosis. IOSR J Dent Med Sci. 2013;8(5):05-08. doi: 10.9790/0853-0850508

4. Archana ARK, Sajjan AC, Rao RK, Archana B, Swetha G. A Comparative Study of Auramine Staining Using Light Emitting Diode (LED) Fluorescent Microscopy with Ziehl-Neelsen Staining for Detection of Acid Fast Bacilli in Sputum in a Tertiary Care Hospital, Karimnagar. Int J Curr Microbiol Appl Sci. 2018;7(9):117-123. doi: 10.20546/ijcmas.2018.709.015

5. Dzodanu EG, Afrifa J, Acheampong DO, Dadzie I. Diagnostic yield of fluorescence and Ziehl-Neelsen staining techniques in the diagnosis of pulmonary tuberculosis: a comparative study in a District Health Facility. Tuberculosis Research and Treatment. 2019;2019:4091937. doi: 10.1155/2019/4091937

6. Workineh M, Maru M, Seman I, et al. Agreement between direct fluorescent microscopy and ZiehlNeelsen concentration techniques in detection of pulmonary tuberculosis in northwest Ethiopia. Ethiop J Health Sci. 2017;27(5):459-464. doi: 10.4314/ejhs. v27i5.3

7. Imaz M, Allassia S, Aranibar M, et al. Performance of LED fluorescence microscopy for the detection of acid-fast bacilli from respiratory samples in peripheral laboratories in Argentina. Biomedica. 2017;37(2):164174. doi: 10.7705/biomedica.v37i2.3276

8. Hanscheid T. The future looks bright: lowcost fluorescent microscopes for detection of Mycobacterium tuberculosis and Coccidiae. Trans R Soc Trop Med Hyg. 2008;102(6):520-521. doi: 10.1016/j. trstmh.2008.02.020

9. Ba F, Rieder HL. A comparison of fluorescence microscopy with the Ziehl-Neelsen technique in the examination of sputum for acid-fast bacilli. Int $J$ Tuberc Lung Dis. 1999;3(12):1101-1105. PMID: 10599014.

10. Shuaib YA, Khalil EAG, Schaible UE, et al. Smear Microscopy for Diagnosis of Pulmonary Tuberculosis in Eastern Sudan. Tuberc Res Treat. 2018;2018:8038137. doi: $10.1155 / 2018 / 8038137$

11. Kivihya-Ndugga LEA, Van Cleeff MRA, Githui WA, et al. A comprehensive comparison of Ziehl-Neelsen and fluorescence microscopy for the diagnosis of tuberculosis in a resource-poor urban setting. The

\section{ETHICS STATEMENT}

Ethical clearance was obtained from the 
Int J Tuberc Lung Dis. 2003;7(12):1163-1171. PMID: 14677891

12. National Tuberculosis Elimination Programme. 2020 https://tbcindia.gov.in/WriteReadData/NSP\%20 Draft\%2020.02.2017\%201.pdf

13. Jadhav DS, Deshmukh VV, Mahato NH, Valand AG. Comparative Study of Ziehl-Neelsen Stain versus Fluorescent Microscopy in Diagnosis of Tuberculous Lymphadenitis on FNAC at A Tertiary Care Centre. Trop J Path Micro. 2019;5(4):217-222. doi: 10.17511/ jopm.2019.i04.06

14. Lawrence, Debbarma M, Baveja CP, Kumar S, Khanna A, Sapriina J. Comparative evaluation of flourescent staining with ziehl-neelsen and kinyoun staining in the diagnosis of clinically suspected cases of pulmonary tuberculosis. International Journal of Contemporary Medical Research. 2016;3(7):1970-1974.

15. Makeshkumar V, Madhavan R, Narayanan S. Prevalence of drug resistance in Mycobacterium tuberculosis in a teaching hospital of Kanchipuram District, Tamil Nadu, South India. Am J Microbiol Res. 2014;2(1):35-40. doi: 10.12691/ajmr-2-1-5

16. Laifangbam S, Singh HL, Singh NB, Devi KM, Singh NT. A comparative study of fluorescent microscopy with Ziehl-Neelsen staining and culture for the diagnosis of pulmonary tuberculosis. Kathmandu Univ Med J. 2009;7:226-230. doi: 10.3126/kumj.v7i3.2728

17. Namaji MAA, Maheshkumar S, Nadgir SD. Comparison of Ziehl-Neelsen light microscopy and fluorescent (light-emitting diode) microscopy with solid culture for the diagnosis of tuberculosis with special reference to extrapulmonary cases. Egypt J Chest Dis Tuberc. 2020;69(4):613-619. doi: 10.4103/ejcdt.ejcdt_153_19

18. Khanna A, Sharma P, Sharma S, Khanna M. Comparison of conventional Ziehl-Neelsen staining with LED Fluorescent staining for pulmonary tuberculosis cases. Indian J Microbiol Res. 2016;3(4):363-367.

19. Singh NP, Parija SC. The value of fluorescence microscopy of auramine stained sputum smears for the diagnosis of pulmonary tuberculosis. Southeast Asian J Trop Med Public Health. 1998;29(4):860-863. PMID: 10772577

20. Bansal R, Sharma PK, Jaryal SC, Gupta PK, Kumar D. Comparison of sensitivity and specificity of $Z N$ and fluorescent stain microscopy with culture as gold standard. Journal of Tuberculosis Research. 2017;5(02):118-128. doi: 10.4236/jtr.2017.52013

21. Golia S, Hittinahalli V, Nirmala AR, Sangeetha KT, Asha SK. A comparative study of auramine staining using led fluorescent microscopy with Ziehl-Neelsen staining in the diagnosis of pulmonary tuberculosis. Journal of Evolution of Medical and Dental Sciences. 2013;2(20):3450-3456. doi: 10.14260/jemds/714

22. Pathrikar TG, Bansal VP, Mulay MV, Ghogare HS. Comparison of Ziehl Neelsen smear microscopy and AFB culture in a resource limited setting from various clinical samples. Int J Health Sci Res. 2020;10(4):46-51.

23. Gupta S, Shenoy VP, Bairy I, Muralidharan S. Diagnostic efficacy of Ziehl-Neelsen method against fluorescent microscopy in detection of acid fast bacilli. Asian Pacific Journal of Tropical Medicine. 2010;3(4):328-329. doi: 10.1016/S1995-7645(10)60079-X 